\title{
Influence of oxygen admixtures on the lifetime of metastable antiprotonic helium atoms
}

\author{
R. Pohl,* F. J. Hartmann, B. Ketzer, C. Maierl, and T. von Egidy \\ Physik Department, Technische Universität München, D-85747 Garching, Germany \\ J. Eades, E. Widmann, ${ }^{\dagger}$ and T. Yamazaki ${ }^{\ddagger}$ \\ CERN, CH-1211 Geneva 23, Switzerland \\ M. Kumakura and N. Morita \\ Institute for Molecular Science, Myodaiji, Okazaki 444, Japan \\ R. S. Hayano, M. Hori, T. Ishikawa, and H. A. Torii \\ Department of Physics, University of Tokyo, 7-3-1 Hongo, Bunkyo-ku, Tokyo 113, Japan \\ I. Sugai \\ Institute for Nuclear Study, University of Tokyo, 3-2-1 Midori-cho, Tanashi, Tokyo 188, Japan \\ D. Horváth \\ Research Institute for Particle and Nuclear Physics, H-1525 Budapest, Hungary \\ (Received 3 June 1998)
}

\begin{abstract}
Two experiments have been performed at the CERN Low Energy Antiproton Ring (LEAR) facility to study the lifetime shortening of metastable antiprotonic helium atoms by the presence of ppm admixtures of oxygen. The change in shape and lifetime of the delayed annihilation time spectra can be reproduced fairly well by fitting a simple four-level model to the data and assuming equal quenching cross sections for all metastable levels. From this we deduce a quenching cross section of $(1.35 \pm 0.03) \times 10^{-15} \mathrm{~cm}^{2}$ at a temperature of $100 \mathrm{~K}$. An analysis of data from a previous experiment gives a value for the quenching cross section at room temperature of $(1.34 \pm 0.07) \times 10^{-15} \mathrm{~cm}^{2}$. Resonant laser deexcitation of the levels $(n, l)=(37,34)$ and $(39,35)$ (the lowest metastable levels of the cascades $v=n-l-1=2$ and 3, respectively) was used to examine the time development of the population of these levels. Quenching cross sections of $(1.32 \pm 0.10)$ $\times 10^{-15} \mathrm{~cm}^{2}$ and $(1.90 \pm 0.10) \times 10^{-15} \mathrm{~cm}^{2}$ were deduced for the $v=2$ and $v=3$ cascades from these studies at $100 \mathrm{~K} .[\mathrm{S} 1050-2947(98) 06110-1]$
\end{abstract}

PACS number(s): 36.10.-k, 34.90.+q, 82.20.Pm

\section{INTRODUCTION}

An antiproton introduced into ordinary matter will usually annihilate with a nucleus within picoseconds. Only one exception is so far known to this general rule: About $3 \%$ of antiprotons stopped in helium can survive up to some tens of microseconds [1-7], an effect that has been ascribed to the formation of long-lived $\bar{p} \mathrm{He}^{+} \equiv \bar{p}-e^{-}-\mathrm{He}^{2+}$ exotic atoms [8-16]. This exotic system is somewhat similar on the one hand to a hydrogenic atom and to a molecule on the other. The term "atomcule" has therefore been invented to express this dual character.

After collisions with ordinary helium atoms slow down the antiproton to some tens of $\mathrm{eV}$, it is captured by a helium atom and replaces one of its electrons. The principal quantum number $n$ of the initially populated states is known have the value $n \sim n_{0}=\sqrt{M^{*} / m_{e}} \simeq 38$, while the orbital quantum

\footnotetext{
*Present address: Paul Scherrer Institut, CH-5232 Villigen PSI, Switzerland.

†Present address: RIKEN, Wako, Saitama 351-01, Japan.

${ }^{\star}$ Present address: Japan Society for the Promotion of Science, 5-3-1 Koji-machi, Chiyoda-ku, Tokyo 102, Japan.
}

number $l$ of the metastable states is large $(l \lesssim n-1)$. Here $M^{*}$ is the reduced mass of the $\bar{p} \mathrm{He}^{2+}$ system and $m_{e}$ is the electron mass.

The metastability of those high- $n$, high- $l$ states is caused by a combination of several effects. The level spacing is around $2 \mathrm{eV}$, which is small compared to the ionization energy of the remaining electron $(\sim 25 \mathrm{eV})$. This causes a strong suppression of fast Auger deexcitation as this would require $|\Delta l|>3$ for the nearly circular orbits. Furthermore, the remaining electron stabilizes the system against annihilation via collisional Stark mixing by removing the $l$ degeneracy of levels with the same $n$. In addition, the electron repels the surrounding helium atoms due to Pauli blocking. Hence the dominant mechanism for deexcitation is through slow radiative transitions. These follow a "propensity" rule $\Delta n=\Delta l=-1[11,12]$.

Figure 1 shows the level scheme of the $\bar{p} \mathrm{He}^{+}$system as obtained by calculations [11-15] and confirmed by experimental results [17-24]. Antiprotons initially captured in metastable levels cascade down along the arrows denoting transitions with $\Delta n=\Delta l=-1$ until they reach the levels that proceed to ionized states via fast Auger transitions $(\Delta l=$ -3 ), where the antiprotons annihilate via the Stark effect. 


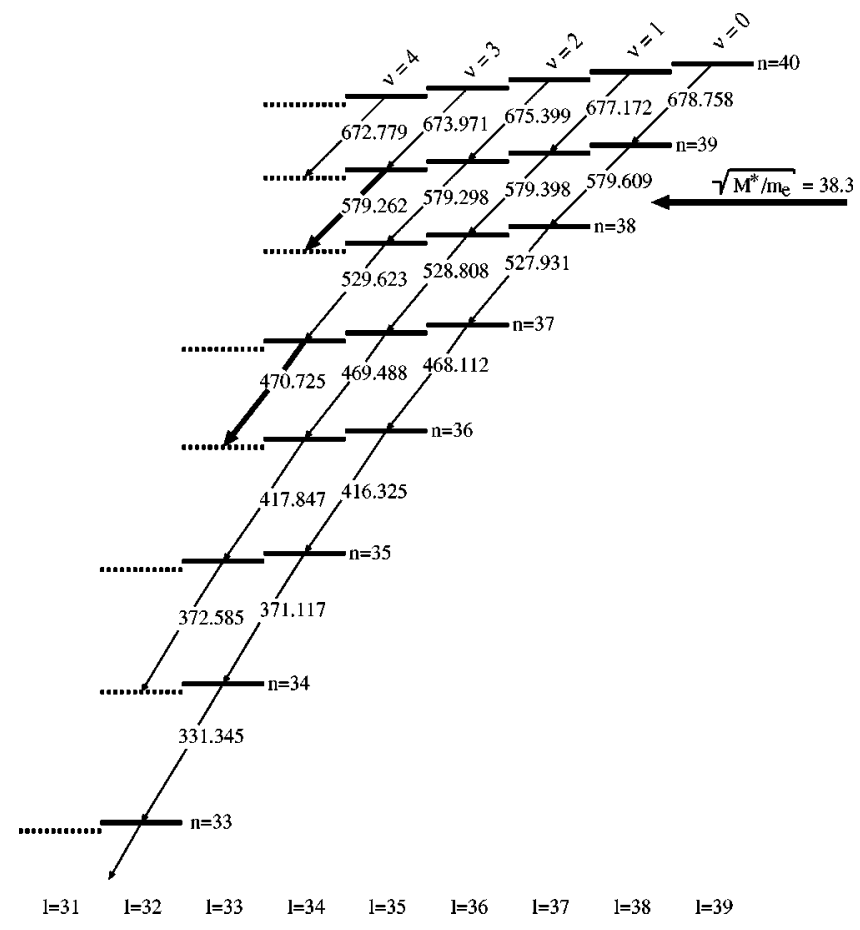

FIG. 1. Part of the level scheme of antiprotonic helium showing the metastable region. Solid lines represent the metastable levels with a lifetime of the order of $1 \mu \mathrm{s}$ and dashed lines indicate shortlived Auger-dominated levels. Transition wavelengths (given in nanometers) are calculated values $[14,15]$.

A delayed annihilation time spectrum (DATS) is the superposition of the contributions of all parallel and nearly independent cascades. As one may imagine, it is impossible to extract information on the initial populations and lifetimes of individual levels from such a spectrum. One could, however, deduce some gross features of the whole metastable region from the DATS analysis.

A precise method for investigating individual levels is to use laser light to induce resonant transitions between two levels [16]. At the end of each metastable cascade with constant $v=n-l-1$ ( $v$ is the radial node number or the vibrational quantum number) a short-lived level exists from which fast Auger deexcitation is possible. If laser light of a frequency appropriate to induce a transition between the last metastable state and the following short-lived state is applied, the antiprotons in the metastable state will almost immediately annihilate. This gives a sharp increase of the annihilation rate in the DATS at the time the laser light pulse arrives in the target gas. The intensity of this peak is proportional to the number of antiprotons present in the metastable level at that time. This laser resonance method has been successfully applied and the $v=2$ and 3 cascades are thereby well established [16-21].

Very soon after the discovery and the detailed studies of the longevity effect [1-6] it was found that admixtures of foreign gases to the helium target gas affect the shape as well as the lifetime of the DATS $[2,3,6,7]$. Light noble gases and $\mathrm{N}_{2}$ have a weak quenching effect while $\mathrm{Xe}, \mathrm{H}_{2}$, and $\mathrm{O}_{2}$ act much more violently. Of all gases investigated so far, oxygen is the one with the strongest influence on the DATS [6]. Only a few hundred ppm of $\mathrm{O}_{2}$ are known to destroy the metastability of the antiprotonic helium atoms completely.
The origin of this quenching effect is not yet understood and it is the purpose of the present paper to shed some light on the behavior of metastable $\bar{p} \mathrm{He}^{+}$in the presence of oxygen molecules at low concentrations.

In order to investigate the influence of $\mathrm{O}_{2}$ admixtures on individual levels we applied the laser resonance technique to the transitions at the end of the $v=2$ and $v=3$ cascade in $\bar{p} \mathrm{He}^{+}$, as has been done in the case of $\mathrm{H}_{2}$ admixtures $[24,25]$. The corresponding metastable levels are characterized by the quantum numbers $(n, l)=(37,34)$ and $(39,35)$ and can be deexcited to the states $(n, l)=(36,33)$ and $(38,34)$ by laser light of $470.724-\mathrm{nm}$ and 597.259 -nm wavelength, respectively.

In Sec. II of this paper we give a short overview of the quenching of metastable $\bar{p} \mathrm{He}^{+}$by foreign gas admixtures. The experimental setup is described in Sec. III. Section IV deals with the analysis of the delayed annihilation time spectra, Section V is dedicated to the laser experiments. Some concluding remarks are given in Sec. VI.

\section{QUENCHING BY FOREIGN GASES}

A simple phenomenological model can be used to describe the quenching of antiprotonic helium by foreign gases $[3,6,7]$. Assuming that the quenching takes place in binary collisions between the antiprotonic helium atom and the foreign gas molecule only after the formation and thermalization of the $\bar{p} \mathrm{He}^{+}$system, the decay rate of a single level can be written as

$$
\lambda_{i}^{\text {total }}=\lambda_{i}^{0}+\lambda_{i}^{q}=\lambda_{i}^{0}+\sigma_{i} n_{a} v_{\text {th }} .
$$

Here $\lambda_{i}^{0}$ and $\lambda_{i}^{q}$ are the intrinsic decay rate and the quenching rate of level $i$, respectively, $\sigma_{i}$ is the quenching cross section of this level, $n_{a}$ is the number density of the admixed gas, and $v_{\text {th }}$ is the relative thermal velocity between the colliding particles $\left(\mathrm{O}_{2}\right.$ and $\left.\bar{p} \mathrm{He}^{+}\right)$, i.e.,

$$
v_{\mathrm{th}}=\sqrt{\frac{8 k T}{\pi \mu}} .
$$

Here $\mu$ is the reduced mass of the colliding system

$$
\mu=\frac{m_{a} M}{m_{a}+M},
$$

with $m_{a}$ the mass of the admixture atom or molecule and $M$ the mass of the antiprotonic helium atom. At the present condition of $T=100 \mathrm{~K}$ the relative thermal velocity $v_{\text {th }}$ is $7.0 \times 10^{4} \mathrm{~cm} / \mathrm{s}$.

An indication that metastable levels are not uniformly affected by foreign gases was seen in DATS with ppm admixtures of hydrogen $[24,25]$. The selective quenching of some levels by $\mathrm{H}_{2}$ creates a fast component in the DATS while shortening the lifetime of the long-lived part, but leaving its structure unchanged. By adding the appropriate amount of hydrogen to the target gas we were able to shorten the lifetime of individual levels selectively and this allowed us to find a completely different type of laser resonance in antiprotonic helium: The so-called hydrogen-assisted inverse resonance method made it possible to observe transitions be- 
tween two normally metastable levels [22]. More recent investigations determined both the $n$ and the $l$ dependence of the lifetime of a number of levels in the presence of hydrogen [25].

\section{EXPERIMENTAL SETUP}

\section{A. Slow extraction mode}

In order to measure the DATS we used the continuous or "slow extraction" mode of the CERN Low Energy Antiproton Ring (LEAR). Running in a parasitic mode in parallel to other experiments, a typical filling of the storage ring provided us with about $30000 \bar{p} / \mathrm{s}$ of $200 \mathrm{MeV} / c$ momentum for about $1 \mathrm{~h}$.

The antiprotons left the beam line, passed a set of scintillation counters, and were stopped in the target gas cooled to $100 \mathrm{~K}$. The products (mostly pions) from the annihilation of the antiproton with a nucleus were detected in seven shower counters (a "sandwich" of scintillators and lead plates) covering a solid angle of about $78 \%$ of $4 \pi$. These counters were able to detect both charged pions and photons from the decay of $\pi^{0}$. The overall probability for the detection of an annihilation was $99.7 \%$ [26].

It could be assumed that if no such annihilation products had been detected within $\sim 100$ ns after the beam counter start signal, a metastable $\bar{p} \mathrm{He}^{+}$atom was present in the target gas. Pre- and postpileup rejection ensured that only one antiproton was present in the target gas at a time, so we were able to measure precisely the time difference between the formation of the $\bar{p} \mathrm{He}^{+}$system and the annihilation of the antiproton with the helium nucleus. The substantial background from slow $\pi^{+} \rightarrow \mu^{+} \rightarrow e^{+}$decays was eliminated via the requirement that at least two shower counters had registered products of the annihilation within $10 \mathrm{~ns}$. Altogether, the slow extraction mode enabled us to record undistorted, background-free spectra of the delayed annihilation of antiprotons in helium with very high efficiency. The disadvantage of this experimental method is, however, that counting single annihilations is rather time consuming and laser spectroscopy in that regime is inefficient.

\section{B. Fast extraction mode}

The second experiment described in this paper used the "fast extraction" mode of the LEAR and a laser to deexcite resonantly individual levels of the antiprotonic helium. In this mode one LEAR filling, which contained about $5 \times 10^{9} \bar{p}$, was used to provide about eight bunches of $200 \mathrm{~ns}$ length. One LEAR stack cycle time was typically $20 \mathrm{~min}$.

One advantage of the use of this mode of the LEAR operation is that a measurement needs less time. One beam bunch creates a spectrum that would take several tens of minutes in the slow extraction mode if the same number of antiprotons were used. Furthermore, in laser experiments one laser firing was enough for an entire bunch of $\bar{p}$, compared to the necessity of a laser shot for each $\bar{p}$ in the slow extraction mode. Even more important, we were able to trigger the extraction of antiprotons from the LEAR from the experiment, so we knew in advance at what time the antiprotons would arrive in our target chamber. This knowledge was of

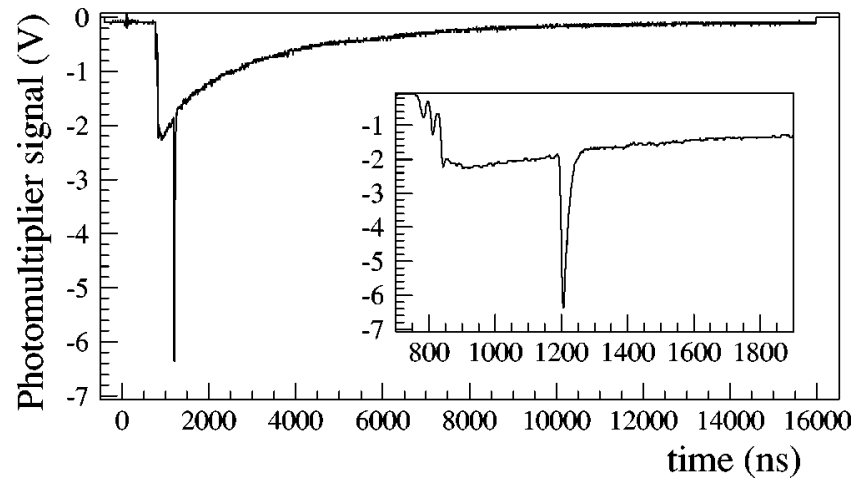

FIG. 2. Analog DATS with laser resonance peak in pure helium taken at $100 \mathrm{~K}, 8$ bars, and $597.259 \mathrm{~nm}$. The inset zooms in on the peak region.

special importance in our laser experiments because the intrinsic delay of our electronics and the laser system itself was about $1.2 \mu \mathrm{s}$. The use of fast extraction enabled us to trigger the laser in advance and consequently to study the population of individual levels as soon as $200 \mathrm{~ns}$ after the formation of $\bar{p} \mathrm{He}^{+}$. In the slow extraction mode, where the antiprotons would arrive randomly, this intrinsic delay had previously limited the earliest laser firing time to $1.2 \mu \mathrm{s}$ after the $\bar{p}$ had stopped in the target.

On the other hand, the high antiproton rate caused difficulties. The method of counting individual annihilations is not applicable, so we used a simple Lucite Čerenkov counter read out by a photomultiplier tube (PMT) to detect the analog sum signal of the pions from many simultaneously annihilating antiprotons [27]. The Cerenkov counter covered a solid angle of about $36 \%$ of $4 \pi$. The negative output voltage of the PMT, which is an analog measurement of the DATS, was recorded with a digital oscilloscope. Figure 2 indicates the high quality of the recorded analog spectra and the laser peak. We also replaced the beam counters with a parallel plate ionization chamber to enable detection of both beam position and profile.

One problem of this experimental method is the presence of a considerable background of $2.2-\mu \mathrm{s}$ lifetime from $\pi^{+}$ $\rightarrow \mu^{+} \rightarrow e^{+}$decay. Assuming that the background is, in first order, proportional to the total number of antiprotons extracted from the beam line, we normalized the laser peak area to the total area to obtain a measure for the population of the deexcited level ("normalized peak intensity"). This way we also eliminated effects caused by variability in the total number of antiprotons arriving in the target and therefore in the total number of $\bar{p} \mathrm{He}^{+}$formed.

Another problem was the following: As only $\sim 3 \%$ of the antiprotons form a metastable $\bar{p} \mathrm{He}^{+}$atom, the signal we were interested in was preceded by a very large peak of prompt annihilations of the remaining 97\%. Earlier experiments with a standard gatable PMT had shown already that this prompt flash of Cerenkov light distorts the delayed signal [27]. We finally obtained the specially designed gated PMT R5504GX-ASSY from Hamamatsu Photonics, which successfully suppressed the prompt peak, completely leaving the signal of the delayed component after 200 ns (nearly) undistorted. 


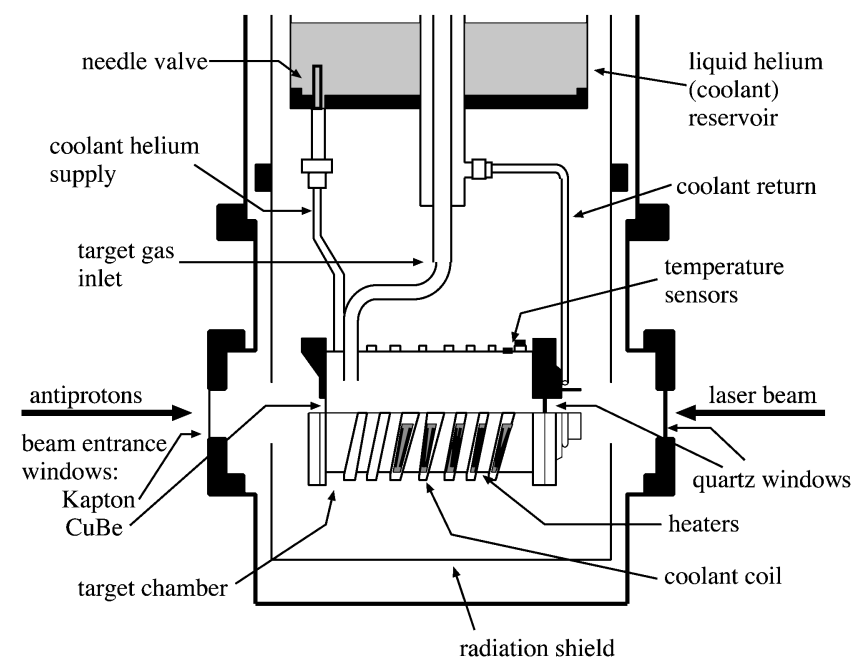

FIG. 3. Target chamber together with the lower part of the cryostat. Antiprotons enter the target chamber from the left through a Kapton and a $\mathrm{CuBe}$ window and are stopped in the target gas. The laser beam can enter the target chamber from the right-hand side through the two quartz windows. An adjustable constant flow of cold helium gas cools the target chamber to cryogenic temperatures, while the heaters allow temperature stabilization with a feedback temperature controller.

\section{Target and laser system}

The target chamber with the helium flow cryostat used to cool the helium target gas to cryogenic temperatures is presented in Fig. 3. Antiprotons entered the target chamber filled with the target gas through the Kapton and the $\mathrm{CuBe}$ window from the left-hand side. Additional Euplex foils were placed in the antiproton beam as degrader material to optimize the $\bar{p}$ stop distribution. A small $\bar{p}$ stopping volume was necessary in the laser experiments to achieve maximum overlap between the created $\bar{p} \mathrm{He}^{+}$and the laser light that entered the chamber through quartz windows on the side opposite to the beam windows.

The target gases used were (a) pure ${ }^{4} \mathrm{He}$ (less than $1 \mathrm{ppm}$ impurity admixtures) and (b) two different mixtures of oxygen $(99.998 \%$ pure) in helium $(99.9996 \%$ pure) at premixed

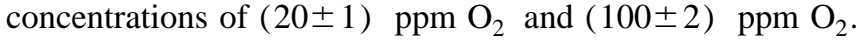

The target chamber could be cooled down to $\sim 5 \mathrm{~K}$ with the help of an adjustable, constant flow of helium through a copper coil that was soldered onto the outer surface of the target chamber wall. Higher temperatures were achieved by heating this coil with the help of several heating wires (35$\mu \mathrm{m}$ stainless steel on 100- $\mu \mathrm{m}$ Kapton) glued onto the coil. The temperature of the target chamber was measured by two sensors and stabilized by a feedback temperature controller. All measurements presented in this paper were performed at $\sim 100 \mathrm{~K}$, which is well above the boiling point of oxygen $(\sim 90 \mathrm{~K})$. This temperature was chosen to ensure that no oxygen could condense inside the cryostat. To ensure a small $\bar{p}$ stopping volume at $100 \mathrm{~K}$ we used high target gas pressures (up to 8 bars).

The laser system we used in the fast extraction experiments consisted of two independent sets of XeCl-excimer laser pumped dye lasers. The excimer laser provided 80-140 $\mathrm{mJ}$ per $\sim 20$-ns pulse. This resulted in a dye laser pulse en-

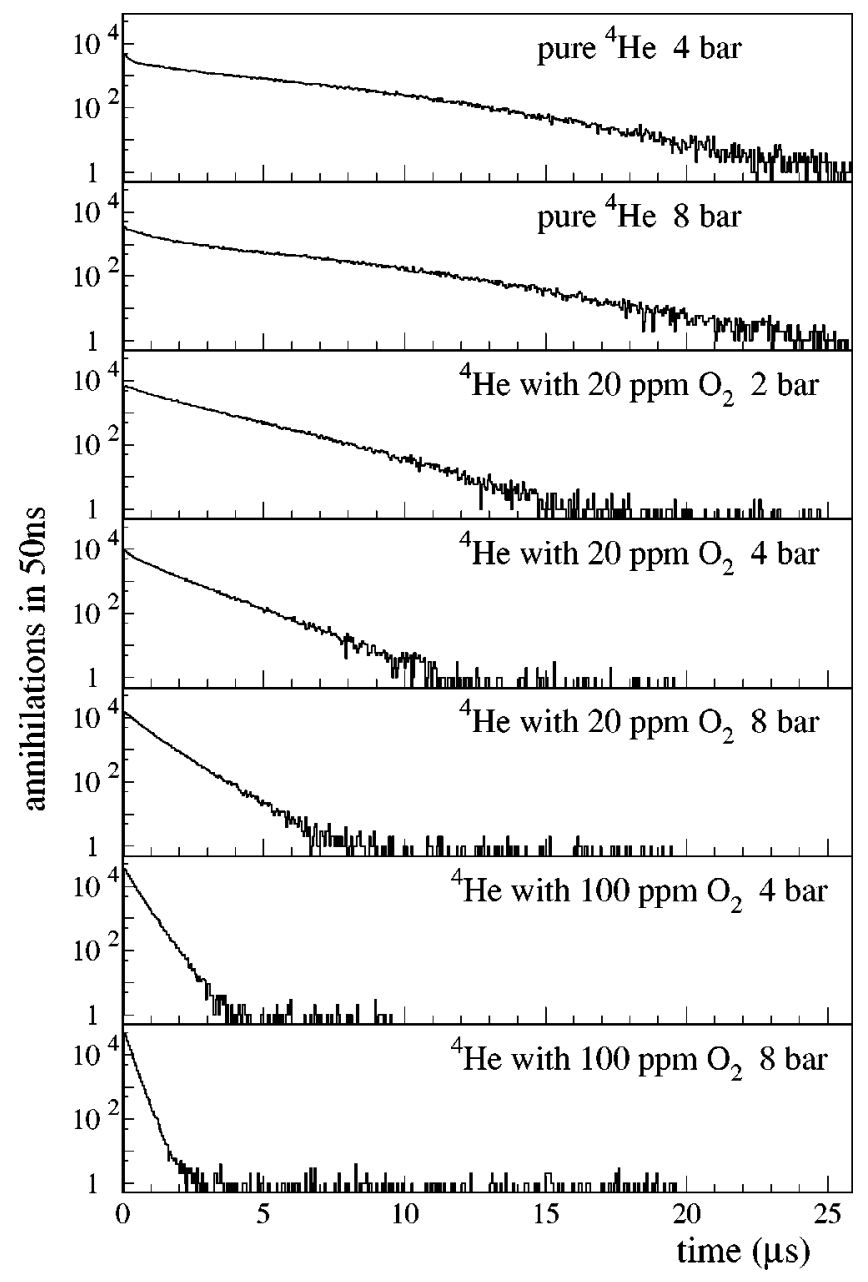

FIG. 4. Delayed annihilation time spectra taken at $100 \mathrm{~K}$ in slow extraction plotted on a semilogarithmic scale, showing that the growth-decay structure in pure $\mathrm{He}$ is reduced to a single exponential when $\mathrm{O}_{2}$ is admixed.

ergy of 2-5 mJ, depending on the wavelength and the dye used. A complete and very detailed description of the experimental setup can be found in another publication [26].

\section{DELAYED ANNIHILATION TIME SPECTRA}

\section{A. Spectra}

The spectra obtained in the slow extraction mode are shown in Fig. 4 and the target conditions are summarized in Table I. Oxygen is, of all gases studied so far, the one that has shown the strongest effect on the DATS [6]. The typical DATS shape seen in pure helium, a fast component followed by a growth-decay structure and a downward bent end, is converted into a nearly single exponential even at very small $\mathrm{O}_{2}$ concentrations. Already an $\mathrm{O}_{2}$ concentration of $3 \times 10^{16} \mathrm{~cm}^{-3}$, which corresponds to a $1 \%$ admixture of a 1-bar room-temperature gas, destroys the metastability of the antiprotonic helium atoms nearly completely. The quenching effect of other gases examined so far ranges from ten times less violent $\left(\mathrm{H}_{2}\right)$, over a few hundred times $\left(\mathrm{N}_{2}\right.$ and $\left.\mathrm{Xe}\right)$, to more than ten thousand times $(\mathrm{Kr}, \mathrm{Ar}$, and $\mathrm{Ne})$ less violent than the effect of oxygen $[6,7]$. 
TABLE I. Summary of the conditions during the DATS measurements at $100 \mathrm{~K}$ in slow extraction and average lifetime $T_{\mathrm{av}}$ of the DATS with and without oxygen admixtures.

\begin{tabular}{lcccccc}
\hline \hline Spectrum & $\begin{array}{c}\mathrm{O}_{2} \text { admixture } \\
(\mathrm{ppm})\end{array}$ & $\begin{array}{c}\text { Pressure } \\
(\text { bars })\end{array}$ & $\begin{array}{c}\text { Temperature } \\
(\mathrm{K})\end{array}$ & $\begin{array}{c}\mathrm{O}_{2} \text { density } \\
\left(10^{15} \mathrm{~cm}^{-3}\right)\end{array}$ & $\begin{array}{c}t_{\max } \\
(\mu \mathrm{s})\end{array}$ & $\begin{array}{c}T_{\mathrm{av}}\left(50 \mathrm{~ns}, t_{\max }\right) \\
(\mu \mathrm{s})\end{array}$ \\
\hline pure ${ }^{4} \mathrm{He}, 4$ bars & 0 & $4.042 \pm 0.011$ & $101.1 \pm 0.2$ & 0.0 & 25 & $3.753 \pm 0.011$ \\
pure ${ }^{4} \mathrm{He}, 8$ bars & 0 & $8.032 \pm 0.042$ & $100.1 \pm 0.1$ & 0.0 & 25 & $3.507 \pm 0.012$ \\
$20 \mathrm{ppm} \mathrm{O}_{2}, 2$ bars & $20 \pm 1$ & $2.016 \pm 0.004$ & $100.7 \pm 0.1$ & $2.1 \pm 0.1$ & 15 & $1.895 \pm 0.005$ \\
$20 \mathrm{ppm} \mathrm{O}, 4$ bars & $20 \pm 1$ & $4.064 \pm 0.016$ & $100.2 \pm 0.2$ & $5.9 \pm 0.3$ & 10 & $1.180 \pm 0.004$ \\
$20 \mathrm{ppm} \mathrm{O}_{2}, 8$ bars & $20 \pm 1$ & $7.896 \pm 0.038$ & $100.5 \pm 0.3$ & $11.4 \pm 0.6$ & 7 & $0.695 \pm 0.002$ \\
$100 \mathrm{ppm} \mathrm{O}_{2}, 4$ bars & $100 \pm 2$ & $4.063 \pm 0.003$ & $100.2 \pm 0.1$ & $29.4 \pm 0.6$ & 3.5 & $0.313 \pm 0.001$ \\
$100 \mathrm{ppm} \mathrm{O}_{2}, 8$ bars & $100 \pm 2$ & $7.862 \pm 0.037$ & $100.0 \pm 0.4$ & $57.0 \pm 1.2$ & 2 & $0.172 \pm 0.001$ \\
\hline \hline
\end{tabular}

\section{B. Average DATS decay time}

A simple way to analyze the DATS behavior under the influence of foreign gas admixtures is to determine an average lifetime $T_{\mathrm{av}}$ of the time spectra

$$
T_{\mathrm{av}}\left(t_{0}, t_{\max }\right)=\frac{\int_{t_{0}}^{t_{\text {max }}} t N(t) d t}{\int_{t_{0}}^{t_{\max }} N(t) d t}-t_{0} .
$$

The values for $T_{\text {av }}$ are summarized in Table I, and Fig. 5 reveals a linear relation between $1 / T_{\mathrm{av}}$ and the oxygen admixture. By interpreting the inverse of the average lifetime as an average decay rate, one can then deduce an average quenching cross section of $(1.40 \pm 0.14) \times 10^{-15} \mathrm{~cm}^{2}$ from these numbers using Eq. (1).

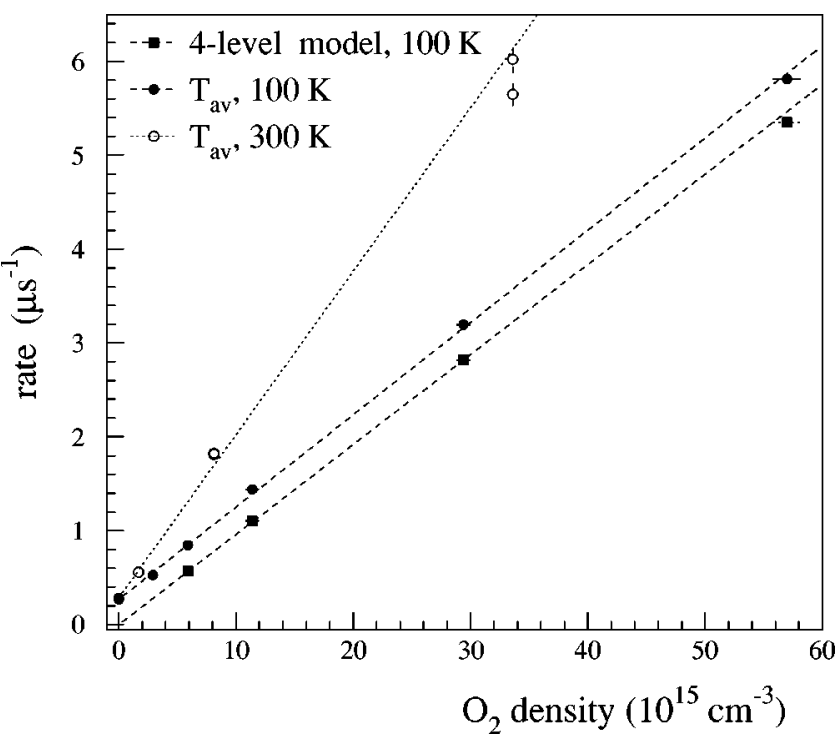

FIG. 5. Result of the DATS analyses with $T_{\text {av }}$ and the four-level model. The inverse of the average lifetime $T_{\mathrm{av}}$ (i.e., average decay rate) and the fitted quenching rate $\lambda_{i}^{q}$ from the four-level model are plotted versus the oxygen number density and the points are fitted with a straight line. At $100 \mathrm{~K}$ the four-level model (solid boxes) and the $T_{\text {av }}$ analysis (solid circles) give nearly the same result for the quenching cross section, so the two dashed lines are nearly parallel. The slope of the dotted line [ $T_{\text {av }}$ analysis of the data at $300 \mathrm{~K}$ (open circles)] is larger due to the higher thermal velocity.
The method of analysis using $T_{\mathrm{av}}$ has the advantage of model independence. Nevertheless we performed a more detailed analysis, which is described in the following subsection.

\section{Analysis using a four-level model}

As mentioned above, the complexity of the scheme of metastable levels in $\bar{p} \mathrm{He}^{+}$(Fig. 1) is such that an independent fit of all level populations and transition rates is not possible. As described in earlier publications [5-7], one can, however, fit the DATS of pure helium very well using a simplified single-cascade model. The minimum number of levels required to reproduce both the fast-decaying component at early times and the downward-bent structure at later times is four. The rate equations for the simplified four-level model [Fig. 6(a)] are

$$
\begin{gathered}
\frac{d N_{1}}{d t}=-\lambda_{1} N_{1}, \\
\frac{d N_{2}}{d t}=\lambda_{1} N_{1}-\lambda_{2} N_{2}, \\
\frac{d N_{3}}{d t}=\lambda_{2} N_{2}-\lambda_{3} N_{3}, \\
\frac{d N_{4}}{d t}=\lambda_{3} N_{3}-\lambda_{4} N_{4} .
\end{gathered}
$$

The time-dependent level populations $N_{i}(t)$ are obtained by solving this set of equations. Annihilation takes place only from the lowest metastable level for the case of pure helium target gas, so the annihilation rate to be fitted to the DATS of pure helium is
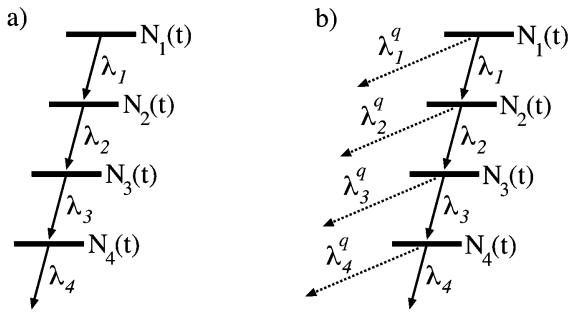

FIG. 6. Four-level model used for the fits of the (a) pure helium DATS and (b) DATS with oxygen admixtures. 


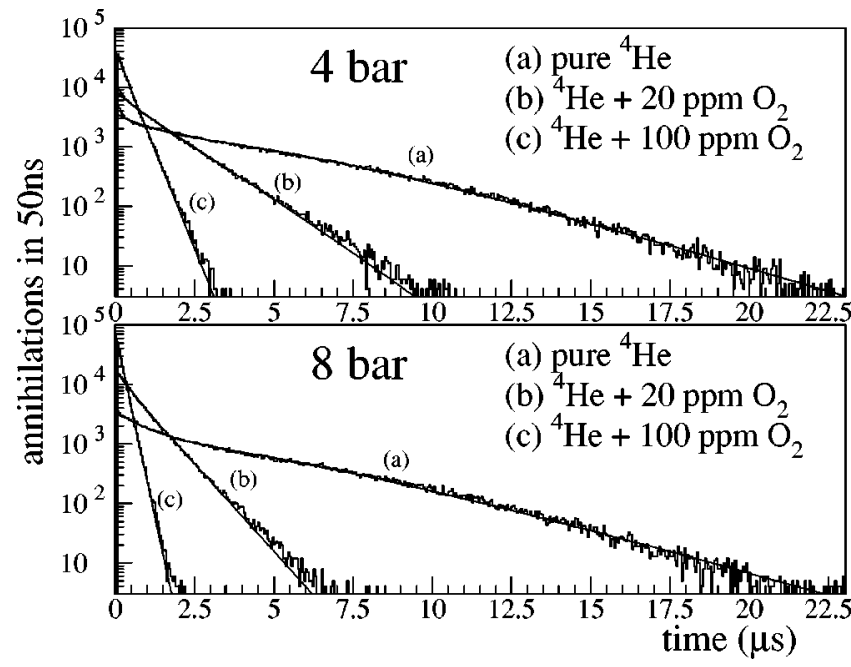

FIG. 7. Typical fits of a four-level model to the DATS assuming equal quenching cross sections for all four levels.

$$
A(t)=\lambda_{4} N_{4}(t)
$$

For the description of the quenching effect of oxygen admixtures we introduce quenching channels to the model [Fig. $6(\mathrm{~b})]$. The rate equations now contain additional quenching terms

$$
\begin{gathered}
\frac{d N_{1}}{d t}=-\left(\lambda_{1}+\lambda_{1}^{q}\right) N_{1}, \\
\frac{d N_{2}}{d t}=\lambda_{1} N_{1}-\left(\lambda_{2}+\lambda_{2}^{q}\right) N_{2}, \\
\frac{d N_{3}}{d t}=\lambda_{2} N_{2}-\left(\lambda_{3}+\lambda_{3}^{q}\right) N_{3}, \\
\frac{d N_{4}}{d t}=\lambda_{3} N_{3}-\left(\lambda_{4}+\lambda_{4}^{q}\right) N_{4} .
\end{gathered}
$$

In this case, annihilation may take place from every level; therefore, the annihilation rate we fitted to the data is now given by

$$
A(t)=\lambda_{1}^{q} N_{1}(t)+\lambda_{2}^{q} N_{2}(t)+\lambda_{3}^{q} N_{3}(t)+\left(\lambda_{4}+\lambda_{4}^{q}\right) N_{4}(t) .
$$

The analysis procedure started with a fit of the cascade without quenching channels [Eq. (6)] to the DATS of pure helium. Various parametrizations of both initial populations and decay rates were used to reduce the number of free pa-
TABLE II. Results of the best fit of the four-level model to the DATS at $100 \mathrm{~K}$. All initial populations were set equal. Their values represent the overall normalization of the pure helium DATS. The quenching rates were also assumed to be equal for all four levels. All transition rates are given in $\mu \mathrm{s}^{-1}$.

\begin{tabular}{lcc}
\hline \hline Parameter & 4 bars & 8 bars \\
\hline$N_{i}^{0}$ & $2.86 \pm 0.01$ & $2.19 \pm 0.01$ \\
$\lambda_{1}=\lambda_{2}$ & $0.412 \pm 0.002$ & $0.390 \pm 0.002$ \\
$\lambda_{3}$ & $0.640 \pm 0.007$ & $0.811 \pm 0.009$ \\
$\lambda_{4}$ & $1.260 \pm 0.013$ & $1.611 \pm 0.019$ \\
$\lambda_{i}^{q}(20 \mathrm{ppm})$ & $0.570 \pm 0.002$ & $1.102 \pm 0.004$ \\
$\lambda_{i}^{q}(100 \mathrm{ppm})$ & $2.818 \pm 0.009$ & $5.349 \pm 0.021$ \\
\hline \hline
\end{tabular}

rameters from eight to four. All parametrizations gave fits of similar quality along with similar results for initial populations and decay rates along the cascade.

Afterward we fitted the DATS with $\mathrm{O}_{2}$ admixtures using the cascade with quenching channels [Eq. (8)]. Initial populations and decay rates were fixed to the values obtained in the fit of the pure helium spectrum recorded at the same temperature and pressure. This can be justified under the assumption that any change in the DATS comes from the quenching of the already formed antiprotonic helium atoms as required in our simple quenching model [Eq. (1)].

The single-exponential shape of the DATS with oxygen admixtures suggests a similar quenching cross section for all metastable levels, and in order to reduce the number of free parameters in the fit we decided to use the same quenching rate for all four levels in the cascade. This rather drastic assumption is justified by the quality of the fit.

To perform this fit procedure one needs a set of DATS consisting of one spectrum with pure helium as target gas and one or more spectra with different admixtures of oxygen. All other parameters that may influence the DATS, such as temperature or pressure, had to be the same for the whole set. We took two of those sets, at $100 \mathrm{~K}$ and 4 and 8 bars of total pressure, respectively. Each set consisted of measurements with 0,20 , and 100 ppm oxygen. The spectrum with 20 ppm $\mathrm{O}_{2}$ at 2 bars was not included in the analysis procedure.

\section{Average quenching cross section}

Figure 7 shows a typical result of the fit procedure just described, applied to both sets of DATS. The best-fitted parameters are presented in Table II. The quenching rates were assumed to be equal for all levels. Despite all simplifying assumptions (only one cascade, equal quenching rates) the model fits the data very well.

TABLE III. Average lifetime $T_{\mathrm{av}}$ (defined as described in the text) of DATS taken at room temperature.

\begin{tabular}{lcccccc}
\hline \hline $\begin{array}{l}\text { Total pressure } \\
\text { (bars) }\end{array}$ & $\begin{array}{c}\mathrm{O}_{2} \text { partial pressure } \\
(\mathrm{mbars})\end{array}$ & $\begin{array}{c}\mathrm{O}_{2} \text { admixture } \\
(\mathrm{ppm})\end{array}$ & $\begin{array}{c}n_{\mathrm{O}_{2}} \\
10^{15} \mathrm{~cm}^{-3}\end{array}$ & $\begin{array}{c}t_{\min } \\
(\mu \mathrm{s})\end{array}$ & $\begin{array}{c}t_{\max } \\
(\mu \mathrm{s})\end{array}$ & $\begin{array}{c}T_{\mathrm{av}}\left(t_{\min }, t_{\max }\right) \\
(\mu \mathrm{s})\end{array}$ \\
\hline 2.5 & 0.06 & 25 & 1.68 & 0.25 & 10.05 & $1.785 \pm 0.036$ \\
12.3 & 0.30 & 25 & 8.13 & 0.05 & 3.95 & $0.548 \pm 0.010$ \\
2.5 & 0.30 & 121 & 8.13 & 0.15 & 3.45 & $0.552 \pm 0.013$ \\
10.3 & 1.25 & 121 & 33.6 & 0.05 & 1.45 & $0.166 \pm 0.004$ \\
2.5 & 1.25 & 500 & 33.6 & 0.05 & 1.45 & $0.177 \pm 0.004$ \\
\hline \hline
\end{tabular}




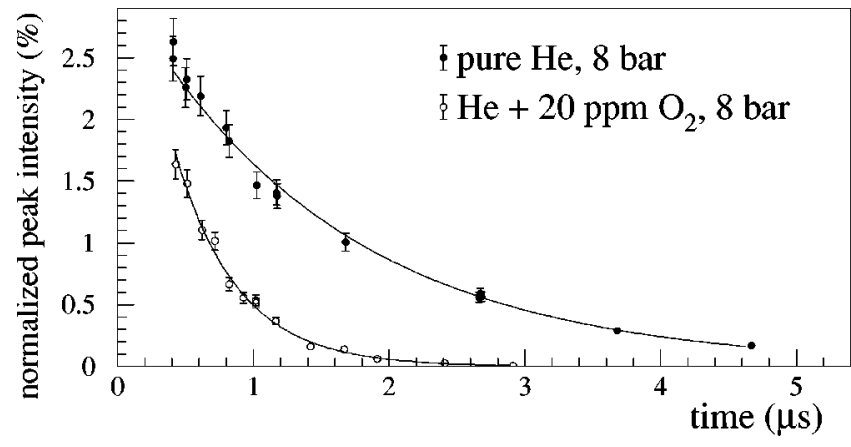

FIG. 8. Laser timing scans of the level $(n, l)=(39,35)$ using the fast extraction mode of LEAR. The normalized peak intensity (i.e., the ratio of the peak area to the total area of the analog spectrum) is plotted versus the laser firing time. Solid and open circles represent data of pure helium and helium with $20 \mathrm{ppm}$ oxygen, respectively; the solid curves show a fit of a single exponential to each set of data. The measurements took place at $100 \mathrm{~K}$ and 8 bars.

Figure 5 gives the quenching rate as a function of oxygen density. It reveals a linear dependence that corroborates the quenching model described. From Eq. (1) we deduced a quenching cross section of $(1.35 \pm 0.03) \times 10^{-15} \mathrm{~cm}^{2}$.

\section{E. Average DATS decay time at room temperature}

The good agreement of the cross sections deduced with the simple method of average lifetime $\left(T_{\mathrm{av}}\right)$ analysis and the ones obtained by fitting a four-level model to the data encouraged us to perform an analysis of DATS with oxygen admixtures obtained in an earlier series of measurements at room temperature [6]. The values for the average lifetimes at room temperature are given in Table III and $1 / T_{\text {av }}$ versus the oxygen number density is plotted in Fig. 5. From Eq. (1) we deduce a quenching cross section of (1.34 \pm $0.07) \times 10^{-15} \mathrm{~cm}^{2}$.

\section{LASER-TAGGED QUENCHING CROSS SECTIONS}

Firing the laser at different times relative to the instant of formation of the metastable antiprotonic helium atoms, we obtained resonance peak intensities $I_{v}(t)$ that (normalized to the total area of the spectrum) give information about the time-dependent $\bar{p}$ population of the deexcited level. We investigated the levels $(n, l)=(37,34)$ and $(39,35)$, the lowest metastable levels of the cascades $v=n-l-1=2$ and 3, using the 470-nm and 597-nm resonances, respectively. Measurements were performed for both levels at $100 \mathrm{~K}, 4$ bars and 8 bars with pure helium gas, 4 bars and 8 bars with 20

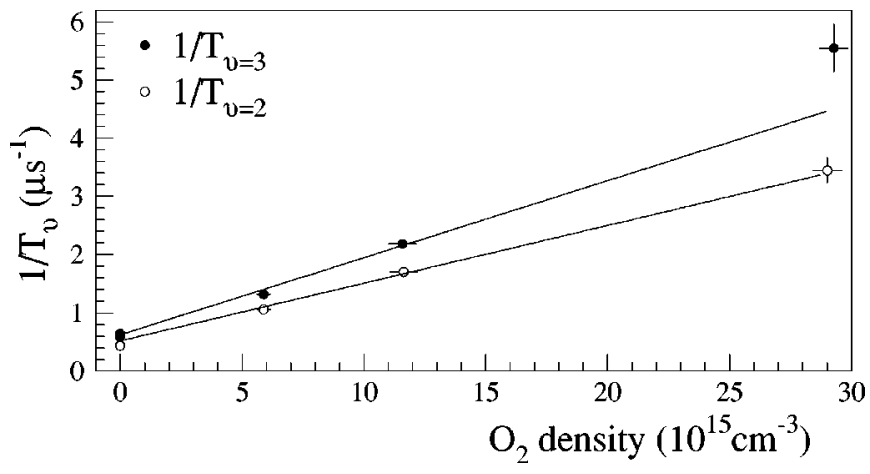

FIG. 9. Single exponential decay rate (i.e., the inverse of the cascade lifetime $T_{v}$ ) versus the $\mathrm{O}_{2}$ density for the two cascades $v=2$ (open circles) and $v=3$ (solid circles).

ppm oxygen admixture, and 4 bars with $100 \mathrm{ppm} \mathrm{O}_{2}$. The statistical treatment of the data is described in Ref. [25].

\section{A. Cascade lifetimes}

The result of a typical laser timing scan, together with a fit of a simple exponential curve

$$
I(t)=I(0) e^{-t / T_{v}},
$$

is shown in Fig. 8. A single exponential decay, however, is a crude approximation, as the time-dependent population of a level is not solely determined by the decay rate of this level. It also depends on feeding from higher levels and hence on the initial populations and decay rates of those higher-lying levels. Nevertheless, we give the values of the "cascade lifetimes" $\left(T_{v}\right)$ of the two cascades as they are model independent (Table IV and Fig. 9). Deducing a quenching cross section using Eq. (1) and identifying its $\lambda_{i}^{\text {total }}$ with $1 / T_{v}$, one finds values of $(1.43 \pm 0.08) \times 10^{-15} \mathrm{~cm}^{2}$ and (1.96 \pm $0.12) \times 10^{-15} \mathrm{~cm}^{2}$ for the cascades $v=2$ and 3 , respectively.

\section{B. Quenching cross sections of the cascades $v=2$ and 3}

In previous publications $[18,19]$ we showed that the $v$ $=2$ and 3 cascades can be described by three and two initially populated metastable levels, respectively. Their initial populations and lifetimes are given in Table V. Using these values we performed a simultaneous fit to all laser timing scans at $470 \mathrm{~nm}(v=2)$ on the one hand and $597 \mathrm{~nm}(v$ $=3$ ) on the other. Each of these two sets of data consisted of five laser timing scans: 4 bars of total pressure with pure helium, 20 and $100 \mathrm{ppm} \mathrm{O}_{2}$ admixture, and 8 bars of total pressure with pure helium and $20 \mathrm{ppm} \mathrm{O}_{2}$.

TABLE IV. Cascade lifetimes $T_{v}$ of the cascades $v=2(470 \mathrm{~nm})$ and $v=3(597 \mathrm{~nm})$ at $100 \mathrm{~K}$ depending on the oxygen number density (given in $10^{15} \mathrm{~cm}^{-3}$ ).

\begin{tabular}{lcccc}
\hline \hline Target gas & $n_{\mathrm{O}_{2}}$ & $T_{v=2}(\mu \mathrm{s})$ & $n_{\mathrm{O}_{2}}$ & $T_{v=3}(\mu \mathrm{s})$ \\
\hline pure ${ }^{4} \mathrm{He}, 4$ bars & 0.0 & $2.32 \pm 0.07$ & 0.0 & $1.73 \pm 0.10$ \\
pure ${ }^{4} \mathrm{He}, 8$ bars & 0.0 & $1.68 \pm 0.04$ & 0.0 & $1.56 \pm 0.04$ \\
$20 \mathrm{ppm} \mathrm{O}_{2}, 4$ bars & $5.9 \pm 0.3$ & $0.944 \pm 0.03$ & $5.9 \pm 0.3$ & $0.758 \pm 0.025$ \\
$20 \mathrm{ppm} \mathrm{O}_{2}, 8$ bars & $11.6 \pm 0.6$ & $0.588 \pm 0.016$ & $11.6 \pm 0.6$ & $0.459 \pm 0.014$ \\
$100 \mathrm{ppm} \mathrm{O}, 4$ bars & $29.0 \pm 0.3$ & $0.290 \pm 0.018$ & $29.3 \pm 0.3$ & $0.180 \pm 0.013$ \\
\hline \hline
\end{tabular}


TABLE V. Initial populations and decay rates used in the fit of the laser data at $100 \mathrm{~K}$. All initial populations and the decay rates of the upper level(s) were fixed to the values in [19] and [18], while the decay rate of the lowest metastable level was fitted to obtain the values $\lambda^{4 \text { bars }}$ and $\lambda^{8 \text { bars }}$ (see the text). The two transition rates denoted by an asterisk were fixed to the theoretical values in [19]. All transition rates are given in $\mu \mathrm{s}^{-1}$.

\begin{tabular}{lclc}
\hline \hline & $v=2(470 \mathrm{~nm})[19]$ & \multicolumn{2}{c}{$v=3(597 \mathrm{~nm})[18]$} \\
\hline$N_{1}^{0}$ & $6 \pm 1$ & $N_{1}^{0}$ & $4.0 \pm 0.3$ \\
$N_{2}^{0}$ & $4 \pm 2$ & $N_{2}^{0}$ & $6.7 \pm 0.7$ \\
$N_{3}^{0}$ & $16 \pm 2$ & & \\
$\lambda_{1}$ & $0.60 \pm 0^{*}$ & $\lambda_{1}$ & $0.49 \pm 0.02$ \\
$\lambda_{2}$ & $0.67 \pm 0^{*}$ & $\lambda_{2}$ & $0.72 \pm 0.02$ \\
$\lambda_{3}$ & $1.16 \pm 0.05$ & & \\
\hline$\lambda_{3}^{4 \text { bars }}$ & $0.75 \pm 0.02$ & $\lambda_{2}^{4}$ bars & $0.88 \pm 0.04$ \\
$\lambda_{3}^{8 \text { bars }}$ & $1.10 \pm 0.03$ & $\lambda_{2}^{8 \text { bars }}$ & $1.09 \pm 0.04$ \\
\hline \hline
\end{tabular}

Each of the two sets $(v=2$ and $v=3)$ was fitted using eight free parameters: To allow for changes in the laser intensity from scan to scan we introduced a normalization parameter for each of the five scans. As we had already established that the lifetime of the lowest metastable level in the $\mathrm{v}=2$ cascade depends very much on the target helium density [28] we introduced two additional parameters $\lambda^{4 \text { bars }}$ and $\lambda^{8 \text { bars }}$. The first is the decay rate of the lowest metastable level for all three scans taken at 4 bars, the other one is used for the two scans at 8 bars. The eighth free parameter is the quenching cross section, which was assumed to be equal for all metastable levels with a common $v$.

Figure 10 shows all laser timing scans taken at $100 \mathrm{~K}$ together with the result of the fits. The data are reproduced very well and the two quenching cross sections for the $v$ $=2$ and 3 cascades obtained from the fit are $(1.32 \pm$ $0.10) \times 10^{-15} \mathrm{~cm}^{2}$ and $(1.90 \pm 0.10) \times 10^{-15} \mathrm{~cm}^{2}$, respectively. These values are in excellent agreement with those obtained from cascade lifetimes $T_{v}$.

\section{CONCLUSIONS}

The DATS analysis shows that a four-level model with equal quenching cross sections for all metastable levels can explain the DATS with and without oxygen admixtures at $100 \mathrm{~K}$ very well, giving a value for the mean quenching cross section at $100 \mathrm{~K}$ of $(1.35 \pm 0.03) \times 10^{-15} \mathrm{~cm}^{2}$.

On the other hand, the value for the quenching cross section at room temperature obtained from $T_{\mathrm{av}}$ analysis applied to DATS is $(1.34 \pm 0.07) \times 10^{-15} \mathrm{~cm}^{2}$, so there is no tem-

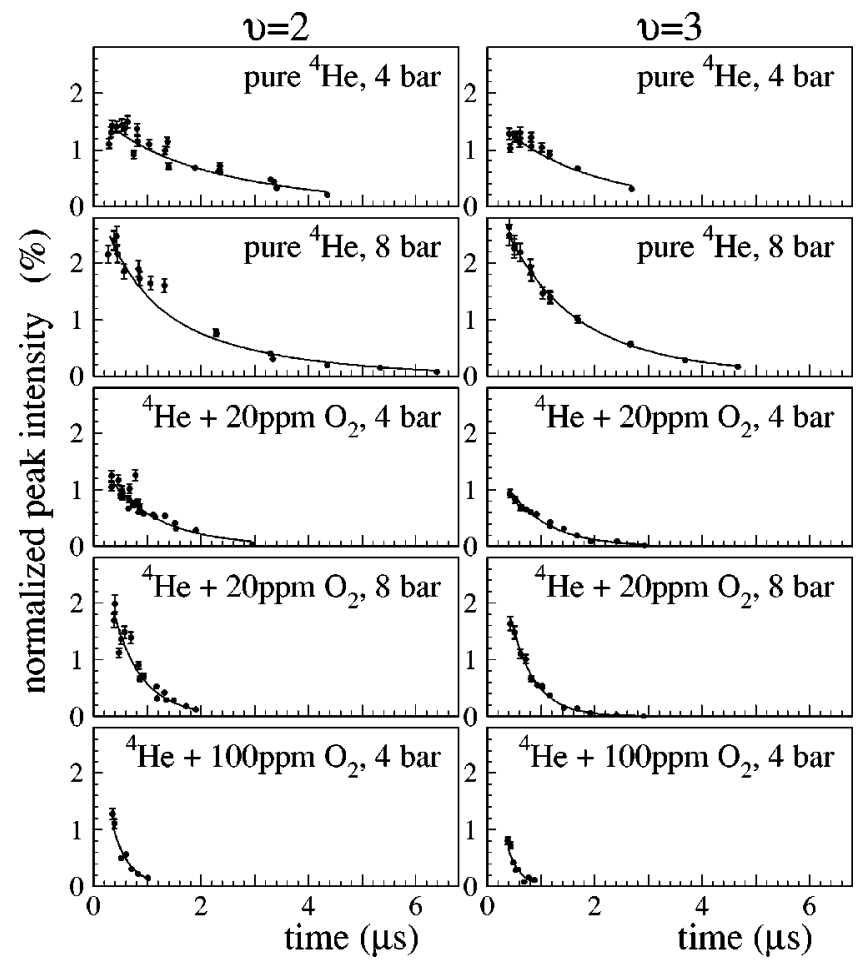

FIG. 10. Laser timing scans with and without oxygen admixture at $100 \mathrm{~K}$ for the levels $(n, l)=(37,34)$ (left) and $(39,35)$ (right). The solid curves are the result of a simultaneous fit of all spectra in a set (see the text).

perature dependence observed between 100 and $300 \mathrm{~K}$.

The laser experiments open a way to study directly the behavior of individual levels and cascades in the presence of small admixtures of oxygen. Here we see a small cascade dependence of the quenching cross section. The cascade $v$ $=2$, whose lowest metastable level is $(n, l)=(37,34)$, is quenched with a cross section of (1.32 \pm $0.10) \times 10^{-15} \mathrm{~cm}^{2}$, whereas the $v=3$ cascade [lowest metastable level $(39,35)]$ has a quenching cross section of $(1.90$ $\pm 0.10) \times 10^{-15} \mathrm{~cm}^{2}$. The effect that levels with higher principal quantum number $n$ are quenched more strongly has already been established for the case of hydrogen [22,24,25], although it is much stronger there. The values for the quenching cross sections are summarized in Table VI.

\section{ACKNOWLEDGMENTS}

We are indebted to the LEAR and PS staffs at CERN for their tireless dedication to providing us with the high-quality

TABLE VI. Summary of the results for the quenching cross sections deduced with different methods. Values are given in $\mathrm{cm}^{2}$.

\begin{tabular}{lcccc}
\hline \hline $\begin{array}{l}\text { Temperature } \\
(\mathrm{K})\end{array}$ & $\begin{array}{c}\text { Analysis } \\
\text { method }\end{array}$ & DATS & \multicolumn{2}{c}{$\begin{array}{c}\text { Laser-tagged cascades } \\
v=2\end{array}$} \\
\hline 100 & $T_{\mathrm{av}}$ & $(1.40 \pm 0.14) \times 10^{-15}$ & $(1.43 \pm 0.08) \times 10^{-15}$ & $(1.96 \pm 0.12) \times 10^{-15}$ \\
100 & four-level model & $(1.35 \pm 0.03) \times 10^{-15}$ & $(1.32 \pm 0.10) \times 10^{-15}$ & $(1.90 \pm 0.10) \times 10^{-15}$ \\
\hline 300 & $T_{\mathrm{av}}$ & $(1.34 \pm 0.07) \times 10^{-15}$ & & \\
\hline \hline
\end{tabular}


antiproton beam, to the CERN cryogenics laboratory (in particular, J.-M. Rieubland) for invaluable help in constructing the high-pressure cryogenic target chamber, and to Hamamatsu Photonics Ltd. for developing the special gated PMT used in the laser experiments. The present work was supported by the German Bundesministerium für Bildung, Wissenschaft, Forschung und Technologie, the Beschleuni- gerlaboratorium der Universität und Technischen Universität München, the Grants-in-Aid for Specially Promoted Research and for International Scientific Research of the Japanese Ministry of Education, Science and Culture, and the Hungarian National Science Foundation (OTKA and OMFB). H.A.T. acknowledges the support of the Japan Society for the Promotion of Science.
[1] M. Iwasaki, S. N. Nakamura, K. Shigaki, Y. Shimizu, H. Tamura, T. Ishikawa, R. S. Hayano, E. Takada, E. Widmann, H. Outa, M. Aoki, P. Kitching, and T. Yamazaki, Phys. Rev. Lett. 67, 1246 (1991).

[2] T. Yamazaki, E. Widmann, R. S. Hayano, M. Iwasaki, S. N. Nakamura, K. Shigaki, F. J. Hartmann, H. Daniel, T. von Egidy, P. Hofmann, Y.-S. Kim, and J. Eades, Nature (London) 361, 238 (1993).

[3] S. N. Nakamura, R. S. Hayano, M. Iwasaki, K. Shigaki, E. Widmann, T. Yamazaki, H. Daniel, T. von Egidy, F. J. Hartmann, P. Hofmann, Y.-S. Kim, and J. Eades, Phys. Rev. A 49, 4457 (1994).

[4] E. Widmann, I. Sugai, T. Yamazaki, R. S. Hayano, M. Iwasaki, S. N. Nakamura, H. Tamura, T. M. Ito, A. Kawachi, N. Nishida, W. Higemoto, Y. Ito, N. Morita, F. J. Hartmann, H. Daniel, T. von Egidy, W. Schmid, J. Hoffmann, and J. Eades, Phys. Rev. A 51, 2870 (1995).

[5] B. Ketzer, F. J. Hartmann, H. Daniel, T. von Egidy, A. Niestroj, S. Schmid, W. Schmid, T. Yamazaki, I. Sugai, K. Nakayoshi, R. S. Hayano, F. E. Mass, H. A. Torii, T. Ishikawa, H. Tamura, N. Morita, D. Horváth, J. Eades, and E. Widmann, Phys. Rev. A 53, 2108 (1996).

[6] E. Widmann, I. Sugai, T. Yamazaki, R. S. Hayano, M. Iwasaki, S. N. Nakamura, H. Tamura, T. M. Ito, A. Kawachi, N. Nishida, W. Higemoto, Y. Ito, N. Morita, F. J. Hartmann, H. Daniel, T. von Egidy, W. Schmid, J. Hoffman, and J. Eades, Phys. Rev. A 53, 3129 (1996).

[7] V. G. Ableev, A. Adamo, M. Agnello, F. Balestra, G. Belli, G. Bendiscioli, A. Bertin, P. Boccaccio, G. C. Bonazzola, T. Bressani, M. Bruschi, M. P. Bussa, L. Busso, D. Calvo, M. Capponi, B. Cereda, P. Cerello, C. Cicalò, M. Corradini, S. Costa, S. De Castro, O. Yu. Denisov, F. D’Isep, A. Donazella, L. Fava, A. Feliciello, L. Ferrero, A. Filippi, V. Filippini, D. Galli, R. Garfagnini, U. Gastaldi, B. Giacobbe, P. Gianotti, A. Grasso, C. Guaraldo, F. Iazzi, A. Lai, A. Lanaro, E. Lodi Rizzini, M. Lombardi, V. Lucherini, A. Maggiora, S. Marcello, U. Marconi, G. V. Margagliotti, G. Maron, A. Masoni, I. Massa, B. Minetti, P. Montagna, M. Morando, L. Musa, F. Nichitiu, D. Panzieri, G. Pauli, M. Piccinini, G. Piragino, M. Poli, S. N. Prakhov, G. Puddu, R. A. Ricci, C. Rizzo, E. Rossetto, A. Rotondi, A. M. Rozhdestvensky, P. Salvini, L. Santi, M. G. Sapozhnikov, N. Semprini Cesari, S. Serci, S. Spighi, P. Temnikov, S. Tessaro, F. Tosello, V. I. Tretyak, G. L. Usai, L. Vannucci, S. Vecchi, G. Vedovato, L. Venturelli, M. Villa, A. Vitale, E. Zavattini, A. Zenoni, A. Zoccoli, and G. Zosi, Nuovo Cimento A 107, 1325 (1994).

[8] G. T. Condo, Phys. Lett. 9, 65 (1964).

[9] J. E. Russell, Phys. Rev. Lett. 23, 63 (1964).
[10] R. Ahlrichs, O. Dumbrajs, H. Pilkuhn, and H. G. Schlaile, Z. Phys. A 306, 297 (1982).

[11] T. Yamazaki, and K. Ohtsuki, Phys. Rev. A 45, 7782 (1992).

[12] I. Shimamura, Phys. Rev. A 46, 3776 (1992).

[13] P. T. Greenland and R. Thürwächter, Hyperfine Interact. 76, 355 (1993); P. T. Greenland (private communication).

[14] V. I. Korobov, Phys. Rev. A 54, R1749 (1996).

[15] V. I. Korobov and D. D. Bakalov, Phys. Rev. Lett. 79, 3379 (1997).

[16] N. Morita, K. Ohtsuki, and T. Yamazaki, Nucl. Instrum. Methods Phys. Res. A 330, 439 (1993).

[17] N. Morita, M. Kumakura, T. Yamazaki, E. Widmann, H. Masuda, I. Sugai, R. S. Hayano, F. E. Maas, H. A. Torii, F. J. Hartmann, H. Daniel, T. von Egidy, B. Ketzer, W. Müller, W. Schmid, D. Horvath, and J. Eades, Phys. Rev. Lett. 72, 1180 (1994).

[18] R. S. Hayano, F. E. Maas, H. A. Torii, N. Morita, M. Kumakura, T. Yamazaki, H. Masuda, I. Sugai, F. J. Hartmann, H. Daniel, T. von Egidy, B. Ketzer, W. Müller, W. Schmid, D. Horvath, J. Eades, and E. Widmann, Phys. Rev. Lett. 73, 1485 (1994); 73, 3181(E) (1994).

[19] F. E. Maas, R. S. Hayano, T. Ishikawa, H. Tamura, A. Torii, N. Morita, T. Yamazaki, I. Sugai, K. Nakayoshi, F. J. Hartmann, H. Daniel, T. von Egidy, B. Ketzer, A. Niestroj, S. Schmid, W. Schmid, D. Horváth, J. Eades, and E. Widmann, Phys. Rev. A 52, 4266 (1995).

[20] H. A. Torii, M. Hori, T. Ishikawa, F. E. Mass, R. S. Hayano, N. Morita, M. Kumakura, I. Sugai, B. Ketzer, H. Daniel, F. J. Hartmann, R. Pohl, R. Schmidt, T. von Egidy, D. Horváth, J. Eades, E. Widmann, and T. Yamazaki, Phys. Rev. A 53, R1931 (1996).

[21] R. S. Hayano, T. Ishikawa, H. Tamura, H. A. Torii, M. Hori, F. E. Mass, N. Morita, M. Kumakura, I. Sugai, F. J. Hartmann, H. Daniel, T. von Egidy, B. Ketzer, R. Pohl, D. Horváth, J. Eades, E. Widmann, and T. Yamazaki, Phys. Rev. A 55, R1 (1997).

[22] B. Ketzer, F. J. Hartmann, T. von Egidy, C. Maierl, R. Pohl, J. Eades, E. Widmann, T. Yamazaki, M. Kumakura, N. Morita, R. S. Hayano, M. Hori, T. Ishikawa, H. A. Torii, I. Sugai, and D. Horváth, Phys. Rev. Lett. 78, 1671 (1997).

[23] T. Yamazaki, E. Widmann, J. Eades, M. Kumakura, N. Morita, H. A. Torii, M. Hori, T. Ishikawa, F. E. Mass, H. Tamura, R. S. Hayano, I. Sugai, Y. Fujita, B. Ketzer, H. Daniel, F. J. Hartmann, M. Hasinoff, R. Pohl, R. Schmidt, T. von Egidy, and D. Horváth, Phys. Rev. A 55, R3295 (1997).

[24] T. Yamazaki, B. Ketzer, E. Widmann, J. Eades, H. Daniel, F. J. Hartmann, M. Hasinoff, R. Pohl, R. Schmidt, T. von Egidy, D. Horváth, M. Kumakura, N. Morita, I. Sugai, Y. Fujita, H. A. Torii, M. Hori, T. Ishikawa, F. E. Mass, H. Tamura, and R. 
S. Hayano, Chem. Phys. Lett. 265, 137 (1997).

[25] B. Ketzer, F. J. Hartmann, T. von Egidy, C. Maierl, R. Pohl, J. Eades, E. Widmann, T. Yamazaki, M. Kumakura, N. Morita, R. S. Hayano, M. Hori, T. Ishikawa, H. A. Torii, I. Sugai, and D. Horváth, J. Chem. Phys. 109, 424 (1998).

[26] H. A. Torii, R. S. Hayano, F. E. Maas, N. Morita, M. Kumakura, T. Yamazaki, H. Masuda, I. Sugai, B. Ketzer, F. J. Hartmann, H. Daniel, T. von Egidy, W. Müller, W. Schmid, D. Horváth, J. Eades, and E. Widmann, Nucl. Instrum. Methods Phys. Res. A 396, 257 (1997).
[27] A. Niestroj, F. J. Hartmann, H. Daniel, B. Ketzer, T. von Egidy, F. E. Mass, R. S. Hayano, T. Ishikawa, H. Tamura, H. A. Torii, N. Morita, T. Yamazaki, I. Sugai, K. Nakayoshi, D. Horváth, J. Eades, and E. Widmann, Nucl. Instrum. Methods Phys. Res. A 373, 411 (1996).

[28] M. Hori, H. A. Torii, R. S. Hayano, T. Ishikawa, F. E. Mass, H. Tamura, B. Ketzer, F. J. Hartmann, R. Pohl, C. Maierl, T. von Egidy, M. Kumakura, N. Morita, I. Sugai, D. Horváth, E. Widmann, J. Eades, and T. Yamazaki, Phys. Rev. A 57, 1698 (1998); 58, 1612(E) (1998); 58, 1613 (1998). 